\title{
SPECTRAL MULTIPLICITY \\ ON PRODUCTS OF HYPERBOLIC SPACES
}

\author{
JONATHAN HUNTLEY
}

(Communicated by William Adams)

\begin{abstract}
Using Fourier expansions and an analysis of the Rayleigh quotient, we obtain an improved upper bound for the dimension of spaces of cusp forms when the symmetric space is a product of upper half spaces. In the rank-one case, some refinements are given.
\end{abstract}

\section{INTRODUCTION AND STATEMENT OF MAIN RESULTS}

In this paper we study two important and related topics in the spectral theory of automorphic forms. The first is the problem of determining conditions on the Fourier coefficients, or Hecke eigenvalues, or local representations of a cusp form that specify it uniquely. The second problem, also of interest in differential geometry, is bounding the multiplicity of eigenvalues of the Laplacian on certain symmetric spaces. Our results on the first problem lead to results on the second.

Throughout this paper, let $H^{n}$ denote hyperbolic $n$-space. Let $\mathscr{H}=H^{n_{1}} \times$ $\cdots \times H^{n_{r}}$ be the product of $r$ hyperbolic spaces. Each $H^{n_{i}}$ is a symmetric space. Let $G_{i}$ denote its group of isometries, and let $G=\prod_{i=1}^{r} G_{i}$ be the group of isometries of $\mathscr{H}$. Let $\Gamma \subset G$ be discrete and such that $\Gamma \backslash \mathscr{H}$ is noncompact but of finite volume. Then $\mathscr{H}$ has a fundamental domain $\mathscr{D}$ with a finite number of cusps. Also, as in the classical case of the upper half plane, if we assume that each $H^{n_{i}}$ is considered in the upper-half-space model, there is, for any given cusp, a $g \in G$ that sends the cusp to infinity.

All of these notions will be explained more fully in $\S 2$. In that section we will also introduce the notion of a cusp form and assign a Fourier expansion to a given cusp form. These are fairly straightforward generalizations of the notions of a Maaß cusp form and its Fourier expansion for the classical modular group. Let $\varphi$ and $\psi$ be two cusp forms, and at the $j$ th cusp, let the Fourier coefficients be $a_{\vec{l}}^{(j)}$ and $b_{\vec{l}}^{(j)}$, respectively. As cusp forms $\phi$ and $\psi$ are eigenfunctions of the ring of $G$-left invariant differential operators on $\mathscr{H}$. The ring may be generated by $r$ operators, all essentially Laplacians. We assume $\phi$ and $\psi$ have

Received by the editors May 13, 1989 and, in revised form, December 15, 1989.

1980 Mathematics Subject Classification (1985 Revision). Primary 11F72; Secondary 58G25.

This work was partially supported by PSC-CUNY Research Foundation grant 6-68342. 
the same eigenvalues, and let $\vec{\lambda}=\left(\lambda_{1}, \ldots, \lambda_{r}\right)$ denote the eigenvalues for the basis operators. We are now in a position to state out main results. For any vector $\vec{\alpha}$ let $|\vec{\alpha}|^{2}=\sum_{i=1}^{k} \alpha_{i}^{2}$.

Theorem 1.1. Let $\Gamma \backslash \mathscr{H}, \phi, a_{\vec{l}}^{(j)}$ and $\vec{\lambda}$ be as above, and let $\Gamma$ be irreducible (see §2). Then there is a constant $C$, associated with $\Gamma$, such that if $a_{\vec{l}}^{(j)}=0$ for all $\vec{l}$ with $|\vec{l}|<C|\vec{\lambda}|^{1 / 2}$, then $\phi=0$.

Theorem 1.2. Let $\Gamma \backslash \mathscr{H}, \phi, \psi, a_{\vec{l}}^{(j)}, b_{\vec{l}}^{(j)}$, and $\vec{\lambda}$ be as above. If $a_{\vec{l}}^{(j)}=b_{\vec{l}}^{(j)}$ for all $\vec{l}$ with $|\vec{l}|<C|\vec{\lambda}|^{1 / 2}$ with $C$ as in Theorem 1.1 , then $\phi=\psi$.

Theorem 1.3. Let $M(\vec{\lambda})$ denote the multiplicity of the space of cusp forms on $\Gamma \backslash \mathscr{H}$ with eigenvalues $\vec{l}$. Then $M(\vec{\lambda})=O\left(|\vec{\lambda}|^{(n-r) / 2}\right)$, where $n=\sum_{i=1}^{r} n_{r}$ is the dimension of the space $\Gamma \backslash \mathscr{H}$.

Theorems 1.2 and 1.3 are corollaries of Theorem 1.1. Theorem 1.3 is striking when $r>1$, as it gives a sharper bound than other techniques. All three theorems will be proved in $\S 2$, but it is useful to describe the ideas behind the proof here. The proofs of the theorems involve the estimation of Rayleigh quotients, generalizing a method of Roeckle [Ro] for estimating from below the first eigenvalue of the modular group. Another account is in [He].

The $x$ variables (see $\S 2$, or for the moment think of the upper half plane $x+i y, y>0$ ) control the maximum multiplicity for a space of cusp forms. The differential equations and periodicity show that a cusp form is determined by its values along a 'hyperplane' with the $y$ values fixed. Using all the automorphy conditions, we show that certain integral inequalities hold uniformly or, more precisely, hold not only for integrals on $\Gamma \backslash \mathscr{H}$ but for integrals with respect to the $x$ variables, with the $y$ variables fixed. We are thus reduced to a lower-dimensional problem. The statements regarding differential equations and periodicity (or more accurately, $\Gamma_{\infty}$ automorphy) hold more generally. For example, they hold for cusp forms on $\mathrm{GL}_{n}(\mathbf{R})$. The procedure used here to show uniformity fails there as it requires an 'honest' Fourier expansion or, more precisely, we need the nilpotent group of the group of isometries to be Abelian. It would be interesting to see if another method can be found.

Both problems have been worked on by many authors. A basic result is the Multiplicity One Theorem. In its modern form, this theorem is proved by Jacquet and Langlands [J-L] for $\mathrm{GL}_{2}$ and by Shalika [Sha] for more general reductive groups. The theorem may be interpreted as saying that knowledge of the Fourier coefficients and eigenvalues of the relevant differential operators determines a cusp form. For the spaces $\mathscr{H}$ considered in this paper, a proof based on the uniqueness of solutions of certain differential equations with prescribed properties can be given.

More recently, various authors have used a variety of techniques to obtain weaker conditions that specify a cusp form. Using properties of Rankin-Selberg $L$-functions, Jacquet, Piatetski-Shapiro, and Shalika [J-P-S] have shown that 
knowing all but a finite number of Fourier coefficients determines a cusp form. No information on differential eigenvalues (or infinity types) is needed in these theorems. In a different direction Moreno [Mo], also using the properties of certain $L$-functions, has shown on $\mathrm{GL}_{n}$ that a suitably chosen finite number of Fourier coefficients determines a cusp form uniquely. The number needed depends on the size of the infinity types. It should be noted that these theorems use techniques that in no way resemble ours.

The problem of determining the multiplicity of the spectrum of the Laplacian on various spaces goes back (at least) to Courant's [Co] improvement of Weyl's asymptotic estimate of the number of eigenfunctions of the Laplacian in a bounded domain. If $N(\lambda)$ denotes the dimension of the space of eigenfunctions of the Laplacian with eigenvalue less than $\lambda$ and $M(\lambda)$ denotes the multiplicity of the space of eigenfunctions with eigenvalue $\lambda$, then $M(\lambda) \leq N(\lambda+\varepsilon)-N(\lambda-\varepsilon)$. So if $N(\lambda)=f(\lambda)+O(g(\lambda))$ then, assuming $g(\lambda)<f(\lambda), M(\lambda)=O(g(\lambda))$.

Work by Gelfand, Piatetski-Shapiro, Borel, and Garland shows that $N(\lambda)$ is finite for all $\lambda$ for quite general spaces. Also, for noncompact spaces $N(\lambda)$ may always equal zero. For rank-one symmetric spaces, the Selberg trace formula ([Sel], [He], [J-L]) can be used to show that if the space has finite volume and dimension $n$, then $M(\lambda)=O\left(\lambda^{(n-1) / 2}\right)$. In some cases this can be improved slightly. For compact symmetric spaces, Varadarajan obtains $N(\lambda) \sim C \lambda^{n / 2}$, along with an error term implying

$$
M(\lambda)=O\left(\lambda^{(n-1) / 2}\right) .
$$

If the space is a product of upper half planes, Efrat $[E]$ obtains

$$
N(\lambda)=C \lambda^{n / 2}+O\left(\frac{\lambda^{(n-1) / 2}}{\log \lambda}\right) .
$$

All of these papers use the trace formula. Seeley [See] uses estimates for the wave operator to obtain

$$
N(\lambda)=C \lambda^{n / 2}+O\left(\lambda^{(n-1) / 2}\right)
$$

for compact manifolds with boundary.

On the other hand, Randol [Ran] has shown that given $A$, there exist spaces with $M(\lambda)>A$ for some $\lambda$. As far as we know, it is unknown whether or not, on a given space such as those we study, $M(\lambda)<A$, where $A$ depends on the space.

The rest of the paper is organized as follows. We begin $\S 2$ by making precise certain concepts mentioned in $\S 1$. We then prove Theorem 1.1. This is the technical heart of the paper. We then prove Theorems 1.2 and 1.3.

In $\S 3$, we consider the rank-one case. We can improve our results in that case. We show in a sense that is made precise, that if sufficiently many Fourier coefficients are 'close', two cusp forms agree. The proof uses an orthogonality argument and the existence of a basis of Hecke eigenforms for the space of cusp 
forms. After this, the argument used is a Rayleigh quotient argument similar to those in $\S 3$. We then mention a case of number-theoretic interest. We close the paper with some explicit results in classical cases.

\section{NOTATION AND PROOFS}

2.1. Notation. As mentioned in $\S 1$, we study in this paper cusp forms on products of hyperbolic spaces. We adopt the following notations:

$$
\mathscr{H}=H^{n_{1}} \times \cdots \times H^{n_{r}},
$$

where $H^{n_{i}}$ is $n_{i}$-dimensional hyperbolic space. As a generalization of the upperhalf-space model of hyperbolic space, we introduce the following coordinates and state several results.

Definition 2.1.2. Coordinates of $\mathscr{H}$ are given by

$$
\left(y_{1}, \ldots, y_{r}, x_{1,1}, \ldots, x_{1, n-1}, \ldots, x_{r, 1}, \ldots, x_{x, n_{r}-1}\right)=(\vec{y}, \vec{x})
$$

with $x_{i, n_{j}} \in \mathbf{R}, y_{i}>0$. If the rank of $\mathscr{H}, r$, is equal to one, then we suppress some subscripts and write $(y, \vec{x})=\left(y, x_{1}, \ldots, x_{n-1}\right)$.

Before introducing the facts that we need in complete generality, we give them when $r=1$. This case, the case of hyperbolic space, is better known, and after seeing the facts here, it is easier to understand the general case. Automorphic forms for rank-one spaces have been studied in many places; for example, see [C-S].

The ring of invariant operators in this case is generated by the Laplacian $\Delta$. In the above coordinates, we have

$$
\Delta \phi=y^{2}\left[\frac{\partial \phi^{2}}{\partial y^{2}}+\sum_{i=1}^{n-1} \frac{\partial \phi^{2}}{\partial x^{2}}\right]-(n-2) y \frac{\partial \phi}{\partial y} .
$$

We will not actually work with $\Delta$ directly. The invariant volume element is given by

$$
d V=\frac{d x_{1} \cdots d x_{n-1} d y}{y^{n}} .
$$

Let $G$ be the isometry group of $\mathscr{H}$. Let $\Gamma \subset G$ be such that $\Gamma \backslash \mathscr{H}$ is noncompact but has finite invariant volume. There is an open set $\mathscr{D} \subset \mathscr{H}$ which satisfies

$$
\bigcup_{\gamma \in \Gamma} \overline{\mathscr{D}}=\mathscr{H}
$$

and

$$
\gamma \mathscr{D} \cap \mathscr{D} \neq \phi \Rightarrow \gamma=I .
$$

Here $\gamma \mathscr{D}$ is the image of the set of points of $\mathscr{D}$ under the action of $\gamma \in \Gamma$, and $I$ is the map sending a point in $\mathscr{H}$ to itself. $\mathscr{D}$ has a finite number of 
cusps, located at $\infty$ or at a point with $y=0$. Given any cusp, there is a $g \in G$ sending the cusp to infinity.

Assume for the moment that only one cusp exists and that it is located at $\infty$. Let $\Gamma_{\infty}$ be the stabilizer of $\infty$. This is a lattice in $\mathbf{R}^{n-1}$. One can find $S \subset \mathscr{H}$ (the Siegel set), such that

$$
\begin{gathered}
S=\left\{(y, \vec{x}) \mid y>a>0, \vec{x} \in \Gamma_{\infty} \backslash \mathscr{H}\right\} \\
S \supset \mathscr{D}, \text { and for a finite set }\left\{\gamma_{i}\right\}_{i=1}^{m}, \gamma_{i} \in \Gamma, \cup_{\gamma_{i}} \gamma_{i} \overline{\mathscr{D}} \supset S .
\end{gathered}
$$

If there are $k$ cusps, $\mathscr{D}$ may be decomposed into $k$ sections, each containing one cusp, and we may, as was already noted, choose $g_{i} \in G$, sending the $i$ th cusp to $\infty$. We may then find $S_{i}$ satisfying (2.1.7) and

$$
S_{i} \supset \mathscr{D}_{i} \text {, and a finite number of copies of } \mathscr{D} \text { cover } S_{i} \text {. }
$$

We are now in a position to define cusp forms. Assume first that $\mathscr{D}$ has one cusp.

Definition 2.1.10. A cusp form is a function $\phi \in L^{2}(\Gamma \backslash \mathscr{H})$ such that

(1) $\Delta \phi=\lambda \phi$

(2) $\int_{\Gamma_{\infty} \backslash \mathscr{H}_{x}} \phi(y, x) d \vec{x}=0 \forall y$, with $\mathscr{H}_{x}$ the projection of $\mathscr{H}$ onto the $x$ variables.

For such functions, a Fourier expansion exists. If $L$ is the lattice corresponding to the action of $\Gamma_{\infty}$, and $L^{*}$ is the dual lattice, we have

$$
\phi(\vec{y}, \vec{x})=\sum_{\overrightarrow{0} \neq \vec{l} \in L^{*}} a_{\vec{l}} y^{(n-1) / 2} K_{s-(n-1) / 2}(2 \pi(n-1)|\vec{l}| y) \exp (2 \pi i(\vec{l} \cdot x)) .
$$

The cuspidal condition 2.1.10 (2) and certain facts about differential equations guarantee that the expansion is in the terms of $K$-Bessel functions. $(s(s-n+1)$ $=\lambda$ here $)$. Finally, if $\nabla$ is the gradient, then

$$
|\nabla \phi|^{2}=y^{2}\left(\left|\frac{\partial \phi}{\partial y}\right|^{2}+\sum_{i=1}^{n-1}\left|\frac{\partial \phi}{\partial x_{i}}\right|\right) \text {. }
$$

It is the gradient, not the Laplacian, that we actually consider in our proof.

Now assume that the rank of $\mathscr{H}$ is $r$. Let $G=G_{1} \times \cdots \times G_{r}$ be the isometry group of $\mathscr{H}$. Each $G_{i}$ is the isometry group of $H^{n_{i}}$. The ring of invariant differential operators is generated by $\Delta_{i}$, the Laplacians on $H^{n_{i}}$. Let $n=\sum_{i=1}^{r} n_{i}$ be the dimension of $\mathscr{H}$. The invariant volume element $d V$ is just $d V_{1} \cdot d V_{2} \cdots d V_{r}$, with $d V_{i}$ the invariant volume element on $H^{n_{i}}$. Let $\Gamma \subset G$ be discrete, with $\Gamma \backslash \mathscr{H}$ noncompact but of finite volume. By saying that $\Gamma$ is irreducible we mean that $\Gamma$ is not commensurate to a group $\Gamma_{a} \times \Gamma_{b}$ with $\Gamma_{a} \subset G_{a}, \Gamma_{b} \subset G_{b}, G_{a}$ and $G_{b}$ nontrivial, and $G=G_{a} \times G_{b}$. As before, a fundamental domain $\mathscr{D}$ exists, as do Siegel sets.

If, for example, $\mathscr{D}$ has one cusp, $S$ has the form

$$
\left\{(\vec{y}, \vec{x}) \mid y_{i}>a_{i}>0, \vec{x} \in \Gamma_{\infty} \backslash H_{x}\right\} .
$$


If there is one cusp, then by a cusp form we mean a function $\phi \in L^{2}(\Gamma \backslash \mathscr{H})$ such that

$$
\begin{gathered}
\Delta_{i} \varphi=\lambda_{i} \phi \\
\int_{\Gamma_{\infty} \backslash H_{x}} \phi(\vec{y}, \vec{x}) d \vec{x}=0 .
\end{gathered}
$$

If there are several cusps, we simply have similar conditions at each cusp.

Again, let $L$ denote the lattice corresponding to the action of $\Gamma_{\infty}$ on $x$ variables, and let $L^{*}$ be its dual lattice. If $\Gamma$ is irreducible, we have a Fourier expansion of the form

$$
\phi(\vec{y}, \vec{x})=\sum_{\overrightarrow{0} \neq \vec{l} \in L^{*}} a_{\vec{l}} f(\vec{x}, \vec{y}) \exp (2 \pi i\langle\vec{l}, x\rangle),
$$

and $f(\vec{l}, \vec{y})$ is essentially a product of $K$-Bessel functions. For example, see [E]. The exact form of the function $f$ is not needed here. A similar expansion is obtainable for each cusp in the more-than-one-cusp case.

For references to the higher-rank case, we refer the reader to [E], [Hum], [Shu], and [Te], and the references therein.

2.2. Proof of Theorem 1.1. Assume, at first, that $\Gamma \backslash \mathscr{H}$ has one cusp. Let $a$ be the minimum of the bottoms of the $y$ variables in the Siegel sets. Let $A$ be the number of copies of $\mathscr{D}$ needed to cover $S$.

Assume, as in the statement of the theorem, that

$$
\Delta \phi_{i}=\lambda_{i} \phi .
$$

The Rayleigh-Ritz method yields

$$
\frac{\int_{\mathscr{Z}} \sum_{i=1}^{r}\left|\nabla_{i} \phi\right|^{2} d V}{\int_{\mathscr{Z}}|\phi|^{2} d V}=\sum_{i=1}^{r} \lambda_{i}
$$

From the Siegel property we have

$$
\frac{\int_{S} \sum_{i=1}^{r}\left|\nabla_{i} \phi\right|^{2} d V}{\int_{S}|\phi|^{2} d V} \leq A \sum_{i=1}^{r} \lambda_{i} \leq A_{1}^{0}|\vec{\lambda}|,
$$

where $A_{1}^{0}$ is easily computed.

The left-hand side of (2.2.2) is less that a constant (depending on the group) times the left-hand side of (2.2.3) because the numerator in (2.2.3) is greater than the numerator in (2.2.2), since the functions are automorphic and $S \supset \mathscr{D}$ but the denominator in (2.2.3) is less than a constant times the denominator in (2.2.2), since a finite number of copies of $\mathscr{D}$ covers $S$. Then

$$
\frac{\int_{a_{1}}^{\infty} \cdots \int_{a_{r}}^{\infty} \int_{\mathscr{Z}_{x}} \sum_{i=1}^{r}\left(\left|\frac{\partial \phi}{\partial y_{i}}\right|^{2}+\left|\frac{\partial \phi}{\partial x_{i}}\right|^{2}\right) d V}{\int_{a_{1}}^{\infty} \cdots \int_{a_{r}}^{\infty}|\phi|^{2} d V} \leq A^{\prime}|\vec{\lambda}|,
$$


with $\mathscr{D}_{x}$ a fundamental domain for the stabilizer of infinity. Replacing $y_{i}$ by the constant $a$ and dropping the positive $\left|\frac{\partial \phi}{\partial y_{i}}\right|^{2}$ terms, we have

$$
\frac{\int_{a_{1}}^{\infty} \cdots \int_{a_{r}}^{\infty} \int_{\mathscr{Z}_{x}} a^{2} \sum_{i=1}^{r}\left|\frac{\partial \phi}{\partial x_{i}}\right|^{2} d V}{\int_{a_{1}}^{\infty} \cdots \int_{a_{r}}^{\infty}|\phi|^{2} d V} \leq A^{\prime}|\vec{\lambda}| .
$$

We now use the Fourier expansion, Fubini's theorem, and the orthogonality to conclude that

$$
\frac{\int_{a_{1}}^{\infty} \cdots \int_{a_{r}}^{\infty} a^{2} \sum_{\vec{l} \neq \overrightarrow{0}}\left|a_{\vec{l}}\right|^{2}|\vec{l}|^{2} f(\vec{l}, \vec{y}) d V}{\int_{a_{1}}^{\infty} \cdots \int_{a_{r}}^{\infty} \sum_{\vec{l} \neq \overrightarrow{0}}\left|a_{\vec{l}}\right|^{2} f(\vec{l}, \vec{y}) d V} \leq A_{1}|\vec{\lambda}|,
$$

or, with $A_{2}$ easy to compute,

$$
\frac{\int_{a_{1}}^{\infty} \cdots \int_{a_{r}}^{\infty} \sum_{\vec{l} \neq \overrightarrow{0}}\left|a_{\vec{l}}\right|^{2}|\vec{l}|^{2} f(\vec{l}, \vec{y}) d V}{\int_{a_{1}}^{\infty} \cdots \int_{a_{r}}^{\infty} \sum_{\vec{l} \neq \overrightarrow{0}}\left|a_{\vec{l}}\right|^{2} f(\vec{l}, \vec{y}) d V} \leq A_{2}|\vec{\lambda}| .
$$

If the $a_{\vec{l}}$ are zero for $|\vec{l}|<A_{2}^{1 / 2}|\vec{\lambda}|^{1 / 2}$, we have a contradiction, proving the theorem.

We now proceed to prove the theorem in the case of several cusps. If there are $k$ cusps, we break $\mathscr{D}$ into $k$ pieces with $\mathscr{D}=\bigcup_{i=1}^{k} \mathscr{D}_{i}$, and after sending the $i$ th cusp to infinity, we take a collection of Siegel sets such that $S_{i} \supset \mathscr{D}_{i}$, with a finite number of copies of $\mathscr{D}$ covering any given $S_{i}$. If each $\mathscr{D}_{i}$ is treated separately, the theorem follows, mutatis mutandis.

2.3. Proofs of Theorems 1.2 and 1.3. Theorem 1.2 is essentially obvious; as the infinity types for $\phi$ and $\psi$ are identical, $\phi-\psi$ is also a cusp form. Hence, its Fourier coefficients satisfy the hypothesis of Theorem 1.1, and the theorem follows.

To prove Theorem 1.3, we make the following observations. First, $\Gamma$ need not be irreducible. If it is

$$
\Gamma \backslash \mathscr{H}=\Gamma_{a} \backslash \mathscr{H}_{a} \times \Gamma_{b} \backslash \mathscr{H}_{b},
$$

and we may work on each piece separately. Second, if Theorem 1.3 is false, then we may, using some linear algebra, construct a function that violates the conclusion of Theorem 1.1, but satisfies the hypothesis, thus creating a contradiction. This is because the number of $|\vec{l}|$ satisfying

$$
|\vec{l}|<A|\vec{l}|^{1 / 2}
$$

is of appropriate size, and this in turn is because $L^{*}$ is a lattice.

\section{SOME REFINEMENT IN THE RANK-ONE CASE}

Assume $r=1$. Let $\phi, \psi$, and all other notations be as in $\S 2$. In addition, assume that an orthogonal basis for the space of cusp forms on $L^{2}(\Gamma \backslash \mathscr{H})$ has 
been chosen, that $\Gamma \backslash \mathscr{H}$ has been chosen, and that $\Gamma \backslash \mathscr{H}$ has only one cusp. The last assumption is only for technical convenience. Let $\phi$ and $\psi$ be elements of this basis. We want to show that if $\phi \neq \psi$, but their early Fourier coefficients are close, most of the $L^{2}$ norm of $\phi-\psi$ arises from the later terms in its Fourier expansion. So assume $\phi \neq \psi$, then

$$
\|\phi-\psi\|^{2}=\|\phi\|^{2}+\|\psi\|^{2}=2 .
$$

Also

$$
\begin{aligned}
\|\phi-\psi\|^{2}= & \int_{\mathscr{C}}|\phi-\psi|^{2} d V \leq C \int_{S}|\phi-\psi|^{2} d V \\
= & C \int_{a}^{\infty} \sum_{\mid \vec{l} \leq \leq N}\left|a_{\vec{l}}-b_{\vec{l}}\right|^{2}\left|K_{s-(n-1) / 2}(2 \pi(n-1)|\vec{l}| y)\right|^{2} d y / y \\
& +C \int_{a}^{\infty} \sum_{|\vec{l}|>N}\left|a_{\vec{l}}-b_{\vec{l}}\right|^{2}\left|K_{s-(n-1) / 2}(2 \pi(n-1)|\vec{l}| y)\right|^{2} d y / y,
\end{aligned}
$$

where $N$ is a parameter to be chosen later, and $a$ depends only on $\Gamma$.

From representation theory, the real part of $s$ takes its values in a compact set. From this we can specify that for a fixed $\Gamma$, all the following integrals satisfy

$$
\int_{a}^{\infty}\left|K_{s-(n-1) / 2}(2 \pi(n-1)|\vec{l}| y)\right|^{2} d y / y<\alpha
$$

since we may uniformly bound the integrand $[\mathrm{He}]$.

From this we conclude that if

$$
\sum_{|\vec{l}| \leq N}\left|a_{\vec{l}}-b_{\vec{l}}\right|^{2}<\frac{1}{3 \alpha}
$$

then

$$
\int_{a}^{\infty} \sum_{|\vec{l}| \leq N}\left|a_{\vec{l}}-b_{\vec{l}}\right|^{2}\left|K_{s-(n-1) / 2}(2 \pi(n-1)|\vec{l}| y)\right|^{2} d y / y>\frac{1}{2}\left(\|\phi\|^{2}+\|\psi\|^{2}\right) .
$$

Condition (3.4) is satisfied if

$$
\left|a_{\vec{l}}-b_{\vec{l}}\right|<\frac{D}{N^{\frac{n-1}{2}}}|\vec{l}|<N,
$$

with $D$ a constant depending on $\Gamma$. However, if $N>E|\vec{\lambda}|^{1 / 2}$ for a suitable constant $E$, we have

$$
\begin{array}{r}
\frac{\|\nabla(\phi-\psi)\|^{2}}{\|\phi-\psi\|^{2}} \geq C \frac{\int_{a}^{\infty} \sum|\vec{l}|^{2}\left|a_{\vec{l}}-b_{\vec{l}}\right|^{2}\left|K_{s-(n-1) / 2}(2 \pi(n-1)|\vec{l}| y)\right|^{2} d y / y}{\int_{a}^{\infty} \sum_{|\vec{l}|>N}\left|a_{\vec{l}}-b_{\vec{l}}\right|^{2}\left|K_{s-(n-1) / 2}(2 \pi(n-1)|\vec{l}| y)\right|^{2} d y / y} \\
\quad \geq 2 C \frac{\int_{a}^{\infty} \sum_{|\vec{l}|>N}|\vec{l}|^{2}\left|a_{\vec{l}}-b_{\vec{l}}\right|^{2}\left|K_{s-(n-1) / 2}(2 \pi(n-1)|\vec{l}| y)\right|^{2} d y / y}{\int_{a}^{\infty} \sum_{|\vec{l}|=N}\left|a_{\vec{l}}-b_{\vec{l}}\right|^{2}\left|K_{s-(n-1) / 2}(2 \pi(n-1)|\vec{l}| y)\right|^{2} d y / y}>|\vec{\lambda}| .
\end{array}
$$

This is a contradiction of our assumption. 
We can state the above discussion as a theorem:

Theorem 3.8. Assume all of the above notations and assumptions. Assume (3.6), or more generally (3.4) holds and $N>E|\vec{\lambda}|^{1 / 2}$. Then $\phi=\psi$.

The case of several cusps can be handled in a similar manner. The techniques needed for the modification are similar to the modifications developed for Theorem 1.1 in the multiple-cusp case.

A variation of the theorem is possible in certain cases of number-theoretic interest. Suppose $n=2$ or $n=3$. For a group $\Gamma$ such as a congruence subgroup of the modular group or the group $\mathrm{SL}_{2}(O)$, where $O$ is the ring of integers of an imaginary quadratic extension of $\mathbf{Q}$, Hecke theory [Mi] provides a natural orthogonal basis, namely the Hecke eigenfunctions. Also, the lattice $L$ essentially corresponds to integers in the number field. In this case it is more convenient to assume the normalization $a_{1}=b_{1}=1$. A result similar to Theorem 3.8 may then be derived. We will show this in a certain case below. It should be noted that some condition guaranteeing orthogonality is needed in order to have theorems of this type. To see this, let $\phi$ and $\psi$ be orthogonal cusp forms with the same infinity type. Let $\varepsilon>0$ be a small number. Then by choosing $\varepsilon$ small enough, $\phi$ and $(1-\varepsilon) \phi+\varepsilon \psi$ will have Fourier coefficients that are very close for a very long time. However, it is obvious that $\phi \neq(1-\varepsilon) \phi+\varepsilon \psi$.

Throughout this paper, the constants mentioned can at least in principle be explicitly calculated. We close the paper with theorems in classical cases where the constants are explicitly computed. This also shows how Hecke theory can be used in these theorems.

Theorem 3.9. Let $\phi$ and $\psi$ be to normalized (even or odd) Hecke eigenforms on $\mathrm{SL}_{2}(\mathbf{Z}) \backslash \mathrm{SL}_{2}(\mathbf{R}) / \mathrm{SO}_{2}(\mathbf{R})$, with $a_{1}=b_{1}=1$ and $\left|a_{n}-b_{n}\right|^{2}<(\sqrt{3} \pi /(4 \sqrt{\lambda})$ for $n<\sqrt{\lambda} \times 2 /(\sqrt{3} \pi)$. Then $\psi=\phi$. (Note that $2 /(\sqrt{3} \pi)=.3675527 \ldots)$. Proof. Let $\varepsilon=\max _{n<N}\left|a_{n}-b_{n}\right|$ with $N$ to be chosen later. We assume, for some $p<\sqrt{\lambda} \times \sqrt{2 / 3} / \pi, a_{n} \neq b_{n}$; otherwise, this is a consequence of Theorem 1.2 , if the constants are computed. So $\phi$ and $\psi$ are orthogonal, and

$$
\|\phi-\psi\|^{2}=\|\phi\|^{2}+\|\psi\|^{2} \text {. }
$$

With $H=\mathrm{SL}_{2}(\mathbf{R}) / \mathrm{SO}_{2}(\mathbf{R})$, we can, using Siegel sets and the fact that fundamental domain for $\Gamma \backslash \mathscr{H}$ is

$$
\mathscr{D}=\left\{(y, x):\left(x^{2}+y^{2}\right)>1,|x|<1 / 2\right\}
$$

and

$$
S=\{(y, x): y>\sqrt{3} / 2,|x|<1 / 2\}
$$

is such that two copies of $\mathscr{D}$ cover $S$, we have

$$
\begin{aligned}
\left.\frac{1}{2} \int_{\sqrt{3} / 2}^{\infty} \sum_{n>0}\left|a_{n}\right|^{2} K_{i r}(2 \pi n y)\right|^{2} d y / y & \geq\|\phi\|^{2} \\
& \geq \frac{1}{4} \int_{\sqrt{3} / 2}^{\infty} \sum_{n>0}\left|a_{n}\right|^{2}\left|K_{i r}(2 \pi n y)\right|^{2} d y / y .
\end{aligned}
$$


In this, $1 / 4+r^{2}=\lambda$. A similar relation holds for $\psi$. These imply

$$
\begin{aligned}
\|\phi-\psi\|^{2} \geq & \frac{1}{4} \int_{\sqrt{3} / 2}^{\infty} \sum_{n>0}\left|a_{n}\right|^{2}\left|K_{i r}(2 \pi n y)\right|^{2} d y / y \\
& +\frac{1}{4} \int_{\sqrt{3} / 2}^{\infty} \sum_{n>0}\left|b_{n}\right|^{2}\left|K_{i r}(2 \pi n y)\right|^{2} d y / y \\
\geq & \frac{1}{2} \int_{\sqrt{3} / 2}^{\infty}\left|K_{i r}(2 \pi n y)\right|^{2} d y / y \equiv \alpha .
\end{aligned}
$$

We also have

$$
\int_{\sqrt{3} / 2}^{\infty}\left|K_{i r}(2 \pi n y)\right|^{2} d y / y=\int_{\sqrt{3} / 2}^{\infty}\left|K_{i r}(2 \pi n t)\right|^{2} d t / t \leq \int_{\sqrt{3} / 2}^{\infty}\left|K_{i r}(2 \pi t)\right|^{2} d t / t .
$$

On the other hand, $\phi-\psi$ also satisfies

$$
\begin{aligned}
\|\phi-\psi\|^{2} \leq & \frac{1}{2} \int_{\sqrt{3} / 2}^{\infty} \sum_{n \leq N}\left|a_{n}-b_{n}\right|^{2}\left|K_{i r}(2 \pi n y)\right|^{2} d y / y \\
& +\frac{1}{2} \int_{\sqrt{3} / 2}^{\infty} \sum_{n>N}\left|a_{n}-b_{n}\right|^{2}\left|K_{i r}(2 \pi n y)\right|^{2} d y / y \\
\leq & \varepsilon^{2} N \alpha+\frac{1}{2} \int_{\sqrt{3} / 2}^{\infty} \sum_{n>N}\left|a_{n}-b_{n}\right|^{2}\left|K_{i r}(2 \pi n y)\right|^{2} d y / y .
\end{aligned}
$$

If $\varepsilon<1 / 2 N$, we have

$$
\int_{\sqrt{3} / 2}^{\infty} \sum_{n>N}\left|a_{n}-b_{n}\right|^{2}\left|K_{i r}(2 \pi n y)\right|^{2} d y / y>\alpha
$$

and

$$
\int_{\sqrt{3} / 2}^{\infty} \sum_{n<N}\left|a_{n}-b_{n}\right|^{2}\left|K_{i r}(2 \pi n y)\right|^{2} d y / y<\alpha
$$

so

$$
\int_{S}|\phi-\psi|^{2} d x d y / y^{2} \leq \int_{\sqrt{3} / 2}^{\infty} \sum_{n>N}\left|a_{n}-b_{n}\right|^{2}\left|K_{i r}(2 \pi n y)\right|^{2} d y / y .
$$

Combining all of this, we have (here $\nabla$ is the Euclidean gradient),

$$
\begin{aligned}
\lambda & =\frac{\int_{\mathscr{X}} y^{2}|\nabla(\phi-\psi)|^{2} d x d y / y^{2}}{\int_{\mathscr{Y}}|\phi-\psi|^{2} d x d y / y^{2}} \\
& \geq \frac{3 \pi^{2}}{4} \frac{\int_{\sqrt{3} / 2}^{\infty} \sum_{n>N}\left|a_{n}-b_{n}\right|^{2} n^{2}\left|K_{i r}(2 \pi n y)\right|^{2} d y / y}{\int_{\sqrt{3} / 2}^{\infty} \sum_{n>N}\left|a_{n}-b_{n}\right|^{2}\left|K_{i r}(2 \pi n y)\right|^{2} d y / y} .
\end{aligned}
$$

If $N^{2}>4 \lambda / 4 \pi^{2}$, we must have $\phi=\psi$.

Finally, we state a similar result. Its proof is similar to the above theorem. See [Hun] for details. 
Theorem 3.20. Let $\Gamma_{0}(N)$ be the Hecke congruence subgroup. Let $\phi$ and $\psi$ be even new forms with eigenvalues $\lambda$. Let $C$ be the number of cusps of the fundamental domain for $\Gamma_{0}(N)$. If in any cusp we have

$$
\left|a_{n}^{i}-b_{n}^{i}\right|<\frac{\sqrt{\pi}}{2} \sqrt[4]{\frac{2}{3 C^{3} N(N+1) \lambda}}
$$

for $n<N \sqrt{2 \lambda C(N+1)} / \pi \sqrt{3}$, then $\phi=\psi$. (Here we assume $a_{1}^{1}=b_{1}^{1}=1$ ).

Of course, we may make this more explicit by noting that

$$
C=\sum_{d \mid N ; d>0} \Phi((d, N / d)) \text {, }
$$

with $\Phi$ Euler's function and $(a, b)$ the greatest common divisor [Shi]. From this we deduce the interesting fact that the space of cusp forms also grows at most as a polynomial in the level.

More explicitly, the number of independent cusp forms if $N$ is prime is $O\left(\sqrt{\lambda} N^{3 / 2}\right)$. This should be compared to Randol's result [Ra], which shows that when $N$ is prime, for infinitely many $\lambda$, we have a multiplicity at at least $(N-1) / 2$.

\section{REFERENCES}

[A] J. Arthur, The trace formula in invariant form, Ann. of Math. 114 (1981), 1-74.

[C] R. Courant, Über die Eigenwerte bie den Differentialgechungen der Mathematischer Physik, Math. Z. 7 (1920), 1-57.

[C-S] P. Cohen and P. Sarnak, Discrete groups and harmonic analysis (to appear).

[E] I. Efrat, Selberg trace formula, rigidity, Weyl's law; and cusp forms, Ph. D. Thesis, New York Univ., 1983.

[Ge] S. Gelbart, Automorphic forms on Adele groups, Ann. of Math. Stud. 83 (1975).

[He] D. Hejhal, The Selberg trace formula for $\operatorname{PSL}_{2}(\mathbf{R})$ II, Lecture Notes in Math., vol. 1001 Springer-Verlag, New York, 1983.

[Hum] J. Humphreys, Arithmetic groups, Lecture Notes in Math., vol. 789, Springer-Verlag, New York, 1980.

[Hun] J. Huntley, Multiplicity one theorems for automorphic forms, Ph. D. Thesis, Stanford, 1987.

[J-L] H. Jacquet and R. Langlands, Automorphic forms on $\mathrm{GL}_{2}$, Lecture Notes in Math., vol. 114, Springer-Verlag, New York, 1970.

[J-P-S] H. Jacquet, I. Pintetski-Shapiro, and J. Shalika, On Euler products and the classification of automorphic representations, I, II, Amer. J. Math. 103 (1987), 499-559, 777-815.

[Mi] T. Miyake, On automorphic forms on $\mathrm{GL}_{2}$ and Hecke operators, Ann. of Math. 94 (1971), 174-189.

[Mo] C. Moreno, An analytic proof of the strong multiplicity one theorem, Amer. J. Math. 107 (1985), 163-206.

[Ran] B. Randol, A remark on the multiplicity of the spectrum of congruence subgroups, Proc. Amer. Math. Soc. 81 (1981), 339-340.

[Ray] J. Rayleigh, On the character of complete radiation at a given temperature, Philos. Mag. 27 (1889).

[Ro] W. Roelcke, Über die Wellengeleichung bei Gronzkreisgruppen ersten Art, Sitzungsber. Heidelb. Akad. Wiss. Math.-Natur. Kl., 1953/1955. 
[See] E. Seeley, An estimate near the boundary for the spectral function of the Laplace operator, Amer. J. Math. 102 (1980), 869-902.

[Sel] A. Selberg, Harmonic analysis and discontinuous groups in weakly symmetric Riemannian spaces with applications to Dirichlet series, J. Indian. Math. Soc. 20 (1956), 47-87.

[Sha] J. Shalika, The multiplicity one theorem for $\mathrm{GL}_{n}$, Ann. of Math. 100 (1974), 171-193.

[Shi] G. Shimura, Introduction to the arithmetic theory of automorphic forms, Princeton Univ. Press, Princeton, NJ, 1971.

[Shu] H. Shimuzu, On discontinuous groups operating on products of upper half planes, Ann. of Math. 77 (1963), 33-71.

[Te] A. Terras, Harmonic analysis on symmetric spaces II, Springer-Verlag, New York, 1988.

Department of Mathematics, Bernard M. Baruch College, City University of New YORK, NEW YORK, NEW YORK 10010 